Finite unions of submodules

\title{
ON FINITE UNIONS OF SUBMODULES
}

\author{
Christian Gottlieb \\ Department of Mathematics, University of Stockholm \\ SE-106 91 Stockholm, Sweden \\ gottlieb@math.su.se
}

\begin{abstract}
This paper is concerned with finite unions of ideals and modules. The first main result is that if $N \subseteq N_{1} \cup N_{2} \cup \cdots \cup N_{S}$ is a covering of a module $N$ by submodules $N_{i}$, such that all but two of the $N_{i}$ are intersections of strongly irreducible modules, then $N \subseteq N_{k}$ for some $k$. The special case when $N$ is a multiplication module is considered. The second main result generalizes earlier results on coverings by primary submodules. In the last section unions of cosets is studied.
\end{abstract}

\section{Introduction}

The Prime avoidance lemma is a well-known and very useful tool in commutative algebra. In it's usual textbook form it reads as follows:

Let $I$ be an ideal, and let $P_{1}, P_{2}, \ldots, P_{n}$ be prime ideals in a commutative ring. Suppose for each $P_{i}$ there is an element in $I$ which is not in $P_{i}$. Then there is in $I$ an element which is in none of the $P_{i}$. 
In other words, if each $P_{i}$ can be avoided by some element in $I$, then there is an element in $I$ which avoids them all. For example the lemma is often used in problems concerning zero-divisors (the set of zero-divisors being a union of prime ideals). Alternatively, we can formulate the Prime avoidance lemma in the positive form:

Suppose $I \subseteq P_{1} \cup P_{2} \cup \cdots \cup P_{n}$. Then $I \subseteq P_{k}$ for some $k$.

This can be generalized in several ways. One can modify the conditions on the $P_{i}$ (for example two of them are allowed to be non-prime). One can consider modules rather than just ideals. Also, one can consider cosets of ideals or modules.

First, let us recall the usual proof of the Prime avoidance lemma. Assuming that the covering $I \subseteq P_{1} \cup P_{2} \cup \cdots \cup P_{n}$ by prime ideals has been reduced so that no $P_{i}$ may be excluded from the union, it has to be proved that $n=1$. So suppose $n>1$. Then there is an element $x$ which lies in $I$ and all $P_{i}, i>1$, but outside $P_{1}$. Indeed to obtain such an element just multiply together elements $x_{k} \in I \cap P_{k} \backslash P_{1}$ for $k>1$. This is where it is used that $P_{1}$ is prime. Now, there is also an element $y$, which lies inside $I$ and $P_{1}$ but outside the other prime ideals. The sum $x+y$ gives the contradiction: $x+y \in I$ but $x+y$ lies outside all $P_{i}$.

This is the most common proof, and a careful look at the argument reveals that it suffices that $n-2$ of the $P_{i}$ are prime. Note that multiplication by elements is used in the proof, so it is not obvious how to generalize to modules.

In my first work on this topic [6], I considered ideals and cosets of ideals. I made a survey of some known results and made some improvements. As a starting-point, I used what I call McCoys lemma, after Neil H. MCoy (see $[12])$ :

If $I=I_{1} \cup I_{2} \cup \cdots \cup I_{s}$ where no $I_{k}$ may be omitted, then any $I_{k}$ contains the intersection of the remaining $I_{j}$. So that for example $I_{2} \cap I_{3} \cap \cdots \cap I_{s}=$ $I_{1} \cap I_{2} \cap \cdots \cap I_{s}$.

Following Stephen McAdam ([11]), I called $I \subseteq I_{1} \cup I_{2} \cup \cdots \cup I_{s}$ an efficient covering by ideals if no $I_{k}$ can be omitted. I will use this terminology also in this paper. Note that it follows immediately from McCoys lemma, that in an efficient covering by more than one ideal, no ideal can be prime. The Prime avoidance lemma follows from this, and this way of arguing is more easily adapted to the case of modules.

McCoy showed that, if the covering is efficient then some power $I^{n}$ is contained in the intersection $I_{1} \cap I_{2} \cap \cdots \cap I_{s}$ (he also showed an analoguos 
result for non-abelian groups). He used this to generalize the Prime avoidance lemma from prime ideals to radical ideals (i.e intersections of prime ideals). In fact he showed that it suffices that all but two of the ideals are radical.

In [6] I discussed the problem of finding the smallest possible $n$ such that $I^{n} \subseteq I_{1} \cap I_{2} \cap \cdots \cap I_{s}$. This problem was solved completely by Jörgen Backelin, [3].

A covering by two ideals $I \subseteq I_{1} \cup I_{2}$ can never be efficient, as is easily seen. A covering by three ideals $I \subseteq I_{1} \cup I_{2} \cup I_{3}$ may be efficient, but then $I_{k} \cap I / I \cap I_{1} \cap I_{2} \cap I_{3}$ is a set of only two elements. In fact, in the general case of an efficient covering by $n$ ideals, the set $I / I \cap I_{1} \cap I_{2} \cap \cdots \cap I_{n}$ is always a finite set. The same holds if $I$ is efficiently covered by cosets of the $I_{i}$. This follows from [13] Lemma 5.2 and is also proved in [7].

In [7] I returned to the subject, this time with focus on finite unions $M=$ $M_{1} \cup M_{2} \cup \cdots \cup M_{n}$ of submodules. I used a module-analogue of McCoys lemma and proved a few theorems given conditions on the $\left(M_{i}: M\right)$ and the radicals $\sqrt{M_{i}: M}$. The main result was that if $M=M_{1} \cup M_{2} \cup \cdots \cup M_{n}$ is an efficient union, and all the $M_{i}$ are primary submodules of $M$, then the ideals $\sqrt{M_{i}: M}$ are all equal to one and the same maximal ideal of the ring ([7] Theorem 9).

Similar results have been obtained by Chin-Pi Lu, [10], and S.E. Atani \& Ü. Tekir, [2]. Lu considers submodules of a module $M$ and coverings $N \subseteq$ $N_{1} \cup N_{2} \cup \cdots \cup N_{n}$ where all but possibly two of the $N_{k}$ are prime submodules of $M$ and where $\left(N_{i}: M\right) \nsubseteq\left(N_{j}: M\right)$, for all $i \neq j$. Then $N \subseteq N_{k}$ for some $k$. This has as a nice application a theorem on the torsion submodule of a finitely generated module over a Noetherian ring ([10] Theorem 3.6).

The avoidance-theorem of $\mathrm{Lu}$ was generalized in [2] to the situation where the $N_{i}$ (with the possible exception of two) are primary submodules of $M$ and $\sqrt{N_{i}: M} \nsubseteq \sqrt{N_{j}: M}$, for all $i \neq j$. I will return to this below in Section 5 and generalize the avoidance-theorems of Lu and Atani-Tekir.

The avoidance-theorem of $\mathrm{Lu}$ has also been generalized by Ahmad Khaksari, who considers a countable union of prime submodules (see [9]).

I would like to mention also the work of P. Quartararo \& H.S. Butts, [14]. They call an ideal a $u$-ideal if it can not be efficiently covered by more than one ideal and a ring a $u$-ring if every ideal in the ring is a $u$-ideal. They prove, among other things, that invertible ideals are $u$-ideals (we will return to this fact in Section 4) and they give the following complete description of $u$-rings. A ring $R$ is a $u$-ring if and only if for each maximal ideal $\mathfrak{m}$ either the residue field $R / \mathfrak{m}$ is infinite or the localization $R_{\mathfrak{m}}$ is a Bézout ring (which 
in the local case means that the ideals are totally ordered). Further research of this kind has been made by F. Callialp \& Ü. Tekir [4].

I have now found it possible to improve the presentation and simultaneously extend some of the earlier results. Thus this paper contains both a survey, or recapitulation, of known results, and also some new results. In order to make the paper self-contained I begin with the very basic ideas.

\section{Basic ideas.}

The most basic idea is the simple fact that the sum of an element inside an ideal and an element outside the same ideal is again an element outside the ideal. And the same holds for modules. This is all you need to prove

Lemma 1. Suppose $N \subseteq N_{1} \cup N_{2}$. Then $N \subseteq N_{1}$ or $N \subseteq N_{2}$.

So a covering by only two modules is never efficient. In the sequel $N$ and $N_{i}$ etc. are always assumed to be submodules of some module $M$ over a commutative ring $R$

Following the basic idea, we can prove the following, which generalizes the McCoy lemma mentioned above.

Proposition 2. Suppose $N \subseteq N_{1} \cup N_{2} \cup \cdots \cup N_{r} \cup N_{1}^{\prime} \cup N_{2}^{\prime} \cup \cdots \cup N_{s}^{\prime}$. Then $N \cap N_{1} \cap N_{2} \cap \cdots \cap N_{r} \subseteq \bigcup_{i=1}^{s} N_{i}^{\prime}$ or $N \cap N_{1}^{\prime} \cap N_{2}^{\prime} \cap \cdots \cap N_{s}^{\prime} \subseteq \bigcup_{i=1}^{r} N_{i}$.

Proof. We give an indirect proof and suppose there is an $x \in N \cap N_{1} \cap N_{2} \cap$. $\cap N_{r} \backslash \bigcup_{i=1}^{s} N_{i}^{\prime}$ and a $y \in N \cap N_{1}^{\prime} \cap N_{2}^{\prime} \cap \cdots \cap N_{r}^{\prime} \backslash \bigcup_{i=1}^{r} N_{i}$. Then $x+y \in N$ but, by the basic idea, $x+y$ is outside every $N_{i}$ and every $N_{i}^{\prime}$.

Usually we will use this in the following special case.

Corollary 3. Suppose $N \subseteq N_{1} \cup N_{2} \cup \cdots \cup N_{s}, s \geq 2$. Then $N \subseteq N_{2} \cup N_{3} \cup \cdots$ $\cup N_{s}$ or $N \cap N_{2} \cap N_{3} \cap \cdots \cap N_{s} \subseteq N_{1}$.

Proof. If $N \cap N_{2} \cap N_{3} \cap \cdots \cap N_{s} \nsubseteq N_{1}$, then according to Proposition 2, $N \cap N_{1} \subseteq N_{2} \cup N_{3} \cup \cdots \cup N_{s}$. But $N \backslash N_{1} \subseteq N_{2} \cup N_{3} \cup \cdots \cup N_{s}$. Thus $N \subseteq N_{2} \cup N_{3} \cup \cdots \cup N_{s}$.

Now it is time to introduce some terminology.

Definition. Let $N \subseteq N_{1} \cup N_{2} \cup \cdots \cup N_{s}$ be a covering of a module $N$ by modules $N_{i}$. If a certain $N_{k}$ can not be omitted (i.e. if $N \nsubseteq \bigcup_{i \neq k} N_{i}$ ), then we shall say that $N_{k}$ is necesary. Thus the covering is efficient if and only if every $N_{k}$ is necessary. 
Let us reformulate Corollary 3.

Corollary 4. Suppose $N \subseteq N_{1} \cup N_{2} \cup \cdots \cup N_{s}, s \geq 2$ and suppose that $N_{1}$ is necessary. Then $N \cap N_{2} \cap N_{3} \cap \cdots \cap N_{s} \subseteq N_{1}$.

Thus a necessary module $N_{k}$ contains the intersection of $N$ and the remaining $N_{i}$.

\section{Strongly irreducible and pseudo-radical modules and ideals.}

To obtain more substantial results, we impose conditions on the submodules, or on the module $M$ itself. We recall the definition of a strongly irreducible submodule.

Definition. A submodule $N$ of $M$ is said to be strongly irreducible (in $M$ ) if whenever $N \supseteq N_{1} \cap N_{2}$, then $N \supseteq N_{1}$ or $N \supseteq N_{2}$ holds.

An example immediately presents itself, namely the prime ideals of a commutative ring. The term strongly irreducible ideal seems to have been coined by W. Heinzer-L. Ratliff-D. Rush. See [8] for a treatise of strongly irreducible ideals. Strongly irreducible modules appear in [1].

Proposition 5. Suppose $N \subseteq N_{1} \cup N_{2} \cup \cdots \cup N_{s}, s \geq 2$ where $N_{1}$ is strongly irreducible. Then $N \subseteq N_{2} \cup N_{3} \cup \cdots \cup N_{s}$ or $N \subseteq N_{1}$. In particular, if $N_{1}$ is necessary, then $N \subseteq N_{1}$.

Proof. If $s=2$, this is just Lemma 1. Suppose $s \geq 3$ and that $N \nsubseteq$ $N_{2} \cup N_{3} \cup \cdots \cup N_{s}$. Then, by Corollary $3, N_{1} \supseteq N \cap N_{2} \cap N_{3} \cap \cdots \cap N_{s}$. Now, if $N_{1} \nsupseteq N$, then, say, $N_{1} \supseteq N_{2}$ and hence $N \subseteq N_{1} \cup N_{3} \cup N_{4} \cup \cdots \cup N_{s}$. Now if $s=3$ the proof is complete (again by Lemma 1). Otherwise, we can go on deleting one of $N_{3}, N_{4}, \ldots, N_{s}$.

We can extend this result to a larger class of modules, which I choose in this paper to call pseudo-radical modules.

Definition. A submodule of $M$, which is the intersection of a family of strongly irreducible submodules of $M$ will be called a pseudo-radical submodule.

Recall that an ideal $I$ is said to be radical if $I=\sqrt{I}$, i.e. if $I$ is an intersection of prime ideals. Thus radical ideals are pseudo-radical. This motivates the terminology.

Proposition 6. Suppose $N \subseteq N_{1} \cup N_{2} \cup \cdots \cup N_{s}, s \geq 2$, where $N_{1}$ is a pseudo-radical submodule of $M$. Then $N \subseteq N_{2} \cup N_{3} \cup \cdots \cup N_{s}$ or $N \subseteq N_{1}$. In particular if $N_{1}$ is necessary, then $N \subseteq N_{1}$.

Proof. Suppose $N \nsubseteq N_{2} \cup N_{3} \cup \cdots \cup N_{s}$ and say $N_{1}=\bigcap_{j \in J} Q_{j}$, where the $Q_{j}$ are strongly irreducible. Then for each $j \in J$ we have $N \subseteq Q_{j} \cup N_{2} \cup N_{3} \cup \ldots$ 
$\cup N_{s}$ and hence $N \subseteq Q_{j}$ follows from Proposition 5. But since this holds for all $j \in J$, we actually have $N \subseteq N_{1}$.

Corollary 7. Suppose $N \subseteq N_{1} \cup N_{2} \cup \cdots \cup N_{s}, s \geq 2$ is an efficient covering. Then none of the $N_{i}$ is pseudo-radical.

I think it is worth-while to formulate Proposition 6 in the special case of rings.

Corollary 8. Suppose $I \subseteq I_{1} \cup I_{2} \cup \cdots \cup I_{s}$ is a covering of an ideal $I$ by ideals $I_{1}, I_{2}, \ldots, I_{s}$ of a commutative ring. Further assume that $I_{1}$ is a pseudo-radical ideal. Then $I \subseteq I_{1}$ or $I \subseteq I_{2} \cup I_{3} \cup \cdots \cup I_{s}$. In particular, if $I_{1}$ is necessary, then $I \subseteq I_{1}$.

Thus in an efficient covering of an ideal by more than two ideals, none of the ideals may be pseudo-radical (in particular they can not be prime). Corollary 7 is all we need to prove a generalization of the Prime avoidance lemma.

Theorem 9. Suppose $N \subseteq N_{1} \cup N_{2} \cup \cdots \cup N_{s}$, where all but possibly two of the submodules $N_{i}$ are pseudo-radical. Then $N \subseteq N_{k}$ holds for some $k$.

Proof. After having reduced the covering to an efficient covering no pseudoradical $N_{i}$ is left. This follows from Corollary 7 . Thus $N$ is covered by the union of just two of the $N_{i}$, and hence by some $N_{k}$ alone.

Example. Suppose $I \subseteq I_{1} \cup I_{2} \cup \cdots \cup I_{s}$, where all but possibly two of the $I_{k}$ are pseudo-radical. Then $I \subseteq I_{k}$ for some $k$. For radical ideals this was proved by McCoy, [12].

Examples. In a valuation ring every ideal is clearly strongly irreducible (the ideals being linearly ordered). In the ring of integers, $Z$, every ideal generated by a prime power is strongly irreducible. Consider the ring $Z \times Z$ and the ideal $I=(4) \times(9)=((4) \times(1)) \cap((1) \times(9))$. Then $I$ is pseudo-radical, but not radical (and not strongly irreducible). We have $\sqrt{I}=(2) \times(3)=$ $((2) \times(1)) \cap((1) \times(3))$.

\section{Coverings in multiplication modules.}

First recall the definition of a multiplication module.

Definition. $M$ is said to be a multiplication module if for every submodule $N$ there is an ideal $I$ such that $N=I M$. Note that it clearly follows that $N=\left(N:{ }_{R} M\right) M$.

Next recall the definition of prime and primary submodules.

Definition. A submodule $N$ of $M$ is said to be a prime submodule of $M$ if whenever $r x \in N$ where $r \in R, x \in M$, either $x \in N$ or $r M \subseteq N$. A 
submodule $N$ of $M$ is said to be a primary submodule of $M$ if whenever $r x \in N$ where $r \in R, x \in M$, either $x \in N$ or $r^{n} M \subseteq N$ for some $n$.

It is not difficult to prove that if $N$ is a prime submodule of $M$, then $(N: M)$ is a prime ideal. Note also that $(N: M)=(N: x)$ for any $x \in M \backslash N$, if $N$ is prime and that $\sqrt{N: M}=\sqrt{N: x}$ for any $x \in M \backslash N$, if $N$ is primary.

Prime modules are not necessarily strongly irreducible, but it is well known and easy to see that prime submodules of multiplication modules are strongly irreducible. Indeed let $N$ be a prime submodule of a multiplication module $M$ and suppose $N \supseteq N_{1} \cap N_{2}$. Then $(N: M) \supseteq\left(N_{1}: M\right) \cap\left(N_{2}: M\right)$ and hence, say, $(N: M) \supseteq\left(N_{1}: M\right)$, where we use that $(N: M)$ is a prime ideal. Therefore $N=(N: M) M \supseteq\left(N_{1}: M\right) M=N_{1}$.

Thus for multiplication modules we have.

Proposition 10. Let $M$ be a multiplication module and suppose we have $N \subseteq N_{1} \cup N_{2} \cup \cdots \cup N_{s}$ a covering by submodules. Suppose that all but possibly two of the $N_{i}$ are intersections of prime submodules. Then $N \subseteq N_{k}$ for some $k$.

Proof. This follows from Theorem 9, since in this case intersections of prime submodules are pseudo-radical.

Quartararo and Butts showed that invertible ideals are $u$-ideals. Following their ideas we can prove the following.

Proposition 11. Suppose $N \subseteq N_{1} \cup N_{2} \cup \cdots \cup N_{s}$ where $N$ is a finitely generated multiplication module such that Ann $N=0$. Then $N \subseteq N_{k}$ for some $k$.

Proof. We use induction on $s$. There are ideals $J_{1}, J_{2}, \ldots, J_{s}$ such that $N \cap N_{i}=J_{i} N$. Thus $N=J_{1} N \cup J_{2} N \cup \cdots \cup J_{s} N$. We may assume that this union is reduced to an efficient union and now we want to prove that $s=1$. Suppose $s \geq 2$. Then $N \neq J_{1} N$ and we obtain from Corollary 4 that $J_{2} N \cap J_{3} N \cap \cdots \cap J_{s} N \subseteq J_{1} N$ and hence $J_{2} J_{3} \cdots J_{s} N \subseteq J_{1} N$. Thus $\left(J_{1}+J_{2} J_{3} \cdots J_{s}\right) N \neq N$, whence it follows that $J_{1}+J_{2} J_{3} \cdots J_{s} \neq R$ and hence that $J_{1}+J_{k} \neq R$ for some $k \geq 2$. Let us say $J_{1}+J_{2} \neq R$ and let $\mathfrak{m}$ be a maximal ideal containing $J_{1}+J_{2}$. We have $N=\left(J_{1}+J_{2}\right) N \cup J_{3} N \cup \cdots \cup J_{s} N$, whence by the induction hypothesis $N=\left(J_{1}+J_{2}\right) N$ and hence $N=\mathfrak{m} N$. But since $N$ is finitely generated it would follow that $N$ is 0 after localizing at $\mathfrak{m}$. But this is impossible since $\operatorname{Ann} N=0$.

Since invertible ideals are finitely generated multiplication ideals with zero annihilator, it follows that invertible ideals are $u$-ideals.

Multiplication modules are indeed very special modules. In the general case we need stronger conditions on the submodules than just being prime. In 
what follows we shall look at conditions on the $\left(N_{i}: N\right)$ or $\left(N_{i}: M\right)$ and generalize the avoidance-theorems of $[10]$ and $[2]$.

\section{Conditions on the ideals $\left(N_{i}: N\right)$ and an avoidance-theorem for primary submodules.}

We need a preparatory lemma before being able to prove the avoidancetheorem for primary submodules.

Lemma 12. Suppose $N \subseteq N_{1} \cup N_{2} \cup \cdots \cup N_{s}, s \geq 3$, where $N_{1}$ and $N_{2}$ are necessary and let $a \in R$ be such that $a N \subseteq N_{3} \cap N_{4} \cap \cdots \cap N_{s}$. Then $a^{2} N \subseteq N_{1} \cap N_{2} \cap \cdots \cap N_{s}$.

Proof. We have $N \subseteq\left(N_{1} \cap N+N_{2} \cap N\right) \cup N_{3} \cup N_{4} \cup \cdots \cup N_{s}$ so, by Corollary 4, $N \cap N_{3} \cap N_{4} \cap \cdots \cap N_{s} \subseteq N_{1} \cap N+N_{2} \cap N$. Thus $a^{2} N \subseteq$ $a\left(N_{1} \cap N+N_{2} \cap N\right)$. Now $a\left(N_{1} \cap N\right) \subseteq N_{1} \cap a N \subseteq N \cap N_{1} \cap N_{3} \cap N_{4} \cap \cdots$ $\cap N_{s}$ but $N_{2} \supseteq N \cap N_{1} \cap N_{3} \cap N_{4} \cap \cdots \cap N_{s}$, since $N_{2}$ is necessary. Thus $a\left(N_{1} \cap N\right) \subseteq N \cap N_{1} \cap N_{2} \cap \cdots \cap N_{s}$. Similarly $a\left(N_{2} \cap N\right) \subseteq N \cap N_{1} \cap N_{2} \cap \cdots$ $\cap N_{s}$. Thus $a^{2} N \subseteq N_{1} \cap N_{2} \cap \cdots \cap N_{s}$.

We now prove a theorem similar of McCoys lemma.

Proposition 13. Suppose $N \subseteq N_{1} \cup N_{2} \cup \cdots \cup N_{s}, s \geq 3$, where $N_{1}$ and $N_{2}$ are both necessary. Then $\sqrt{N_{1}: N} \cap \sqrt{N_{2}: N} \supseteq \bigcap_{i=3}^{s} \sqrt{N_{i}: N}$. In other words $\bigcap_{i=3}^{s} \sqrt{N_{i}: N}=\bigcap_{i=1}^{s} \sqrt{N_{i}: N}$.

Proof. Take any $a \in \bigcap_{i=3}^{s} \sqrt{N_{i}: N}$. Then for some $m$ we have $a^{m} N \subseteq \bigcap_{i=3}^{s} N_{i}$, and hence, by Lemma 12, $a^{2 m} N \subseteq \bigcap_{i=1}^{s} N_{i}$. Thus $a \in \bigcap_{i=1}^{s} \sqrt{N_{i}: N}$.

Corollary 14. Suppose $N \subseteq N_{1} \cup N_{2} \cup \cdots \cup N_{s}, s \geq 3$, is an efficient covering. Suppose also that $\sqrt{N_{1}: N}$ is a prime ideal. Then $\sqrt{N_{1}: N} \supseteq \sqrt{N_{i}: N}$ holds for at least two values of $i \neq 1$.

Proof. By Proposition 13, we have $\sqrt{N_{1}: N} \supseteq \bigcap_{i=3}^{s} \sqrt{N_{i}: N}$. Thus, since $\sqrt{N_{1}: N}$ is prime, $\sqrt{N_{1}: N} \supseteq \sqrt{N_{k}: N}$, some $k \geq 3$. Again, by Proposition 13, $\sqrt{N_{1}: N} \supseteq \bigcap_{i \geq 2, i \neq k} \sqrt{N_{i}: N}$. Thus also $\sqrt{N_{1}: N} \supseteq \sqrt{N_{i}: N}$ for an $i \geq 2$ different from $k$.

Proposition 15. Suppose $N \subseteq N_{1} \cup N_{2} \cup \cdots \cup N_{s}, s \geq 3$, where $\sqrt{N_{i}: N}$ are prime for $i=1,2, \ldots, s-2$. Suppose also that for each $i \leq s-2$, the inclusion $\sqrt{N_{i}: N} \supseteq \sqrt{N_{j}: N}$ holds for at most one $j \neq i$. Then $N \subseteq N_{k}$, some $k$. 
Proof. According to Corollary 14, the covering is not efficient, so we may delete one of the $N_{i}$. As long as $s \geq 3$ we can go on deleting one of the $N_{i}$ without violating the hypotheses. Thus $N$ is contained in the union of two of the $N_{i}$, and hence, by Lemma $1, N \subseteq N_{k}$, some $k$.

Note that we have so far not supposed that the $N_{i}$ are primary. This condition is needed when we focus on $\left(N_{i}: M\right)$ rather than $\left(N_{i}: N\right)$, as in the following theorem which generalizes the main result in [2].

Theorem 16. Suppose $N \subseteq N_{1} \cup N_{2} \cup \cdots \cup N_{s}, s \geq 3$, where $N_{i}$ is a primary submodule of $M$ for $i \leq s-2$. Suppose also that for each $i \leq s-2$ the inclusion $\sqrt{N_{i}: M} \supseteq \sqrt{N_{j}: M}$ holds for at most one $j \neq i$. Then $N \subseteq N_{k}$, some $k$.

Proof. Assume that $N \nsubseteq N_{k}$ for all $k \leq s-2$ and take $x_{k} \in N \backslash N_{k}$ for $k \leq s-2$. Then $\sqrt{N_{k}: M}=\sqrt{N_{k}: x_{k}}=\sqrt{N_{k}: N}$ for $k \leq s-2$. Also $\sqrt{N_{k}: M} \subseteq \sqrt{N_{k}: N}$ for $k=s-1, s$. Thus the hypotheses of Proposition 15 are fulfilled and hence $N \subseteq N_{k}$ for some $k$.

Remark. It follows from [7] Proposition 7 that when $N_{i}$ is a primary submodule, then $\sqrt{N_{i}: M}$ is actually a maximal ideal. As an alternative to the hypotheses in Theorem 16 we could therefore suppose that the $N_{i}$ are primary for $i \leq s-1$ with the $\sqrt{N_{i}: M}, i \leq s-1$ all different.

\section{A few notes on cosets of ideals and modules.}

In this section, we will use the words efficient and necessary in their obvious interpretation for cosets. McCoys lemma can be generalized to cosets in different ways. We shall use the following.

Proposition 17. Suppose $N \subseteq\left(N_{1}+a_{1}\right) \cup\left(N_{2}+a_{2}\right) \cup \cdots \cup\left(N_{s}+a_{s}\right)$ and suppose $N_{1}+a_{1}$ is necessary. Then $N_{1} \supseteq N \cap \bigcap_{i=2}^{s} N_{i}$. In particular if $N_{1}$ is strongly irreducible, then $N \subseteq N_{1}$ (in which case $a_{1} \in N_{1}$ ) or $N_{1} \supseteq N_{k}$, some $k>1$.

Proof. Take any $x \in N \cap \bigcap_{i=2}^{s} N_{i}$ and a $y \in N \cap\left(N_{1}+a_{1}\right) \backslash \bigcup_{i=2}^{s} N_{i}+a_{i}$. Then $x+y \in N \backslash \bigcup_{i=2}^{s} N_{i}+a_{i}$. Thus $x+y \in N_{1}+a_{1}$ and hence $x \in N_{1}$.

Theorem 18. Suppose $N \subseteq\left(N_{1}+a_{1}\right) \cup\left(N_{2}+a_{2}\right) \cup \cdots \cup\left(N_{s}+a_{s}\right)$, where all $N_{i}$ are strongly irreducible and where the modules minimal among $N_{1}$, $N_{2}, \ldots, N_{s}$ each occur only once. Then $\left(N, a_{k}\right) \subseteq N_{k}$, some $k$.

Proof. We may restrict the covering to an efficient one, and after having done so we are supposed to show that $n=1$. Assume $n>1$. Let $N_{k}$ be one of the minimal submodules among the $N_{i}$. Then, by Proposition 17, either 
$N \subseteq N_{k}, a_{k} \in N_{k}$ and there is nothing more to prove, or there is a $j \neq k$ such that $N_{k}=N_{j}$ which would contradict the hypothesis. Thus $\left(N, a_{k}\right) \subseteq N_{k}$ some $k$.

As a corollary we obtain the following result of Gilmer ([5] Lemma 2).

Corollary 19. Suppose $I \subseteq\left(P_{1}+a_{1}\right) \cap\left(P_{2}+a_{2}\right) \cap \cdots \cap\left(P_{r}+a_{r}\right)$, where the $P_{i}$ are pairwise distinct prime ideals. Then $\left(I, a_{k}\right) \subseteq P_{k}$ holds for some $k$.

\section{References.}

1. S. Atani, Strongly irreducible submodules, Bull. Korean Math. Soc. 42 (2005) no. 1, 121-131.

2. S. Atani \& Ü. Tekir, On the primary avoidance theorem for modules over commutative rings, Int. J. Pure Appl. Math. 31 (2006), no.2, 203-207.

3. J. Backelin, Ideal powers in intersections of finite coverings by ideals, Comm. Algebra 22 (1994) no. 8, 3099-3102.

4. F. Callialp \& Ü. Tekir, On unions of prime submodules, Southeast Asian Bull. Math. 28 (2004) no. 2, 213-218.

5. R. Gilmer, Contracted ideals in Krull domains, Duke Math. J. 37 (1970), 769-774.

6. C. Gottlieb, On finite unions of ideals and cosets, Comm. Algebra 22 (1994) no. 8, 3087 - 3097.

7. C. Gottlieb, Modules covered by finite unions of submodules, Comm. Algebra 26 (1998) no. 7, 2351-2359.

8. W. Heinzer, L. Ratliff, D. Rush, Strongly irreducible ideals of a commutative rings., J. Pure Appl. Algebra 166 (2002), no. 3, 267-275.

9. A. Khaksari, On prime avoidance theorem, Int. J. Appl. Math. 20 (2007), no. $1,51-52$.

10. C-P. Lu, Unions of prime submodules, Houston J. Math. 23 (1997), no. 2, 203-213.

11. S. McAdam, Finite coverings by ideals, Ring theory, Proceedings of the Oklahoma conference, Lecture Notes in Pure and Applied Mathematics volume 7, Dekker 1974, 163-171.

12. N. McCoy, A note on finite unions of ideals and subgroups, Proc. Amer. Math. Soc. 8 (1957), 633-637.

13. D.D. Passman, Infinite group rings, Marcel Dekker New York 1971.

14. P. Quartararo Jr. \& H.S. Butts, Finite unions of ideals and modules, Proc. Amer. Math. Soc. 52 (1975), 91-96. 